\title{
Pretérito imperfecto de subjuntivo en el español de Chile: ¿existe alternancia libre entre las desinencias -ra y -se?
}

The Imperfect Subjunctive in Chilean

Spanish: Is There Free Alternation

between -ra and -se Desinences?

\section{María Natalia Castillo Fadić}

Pontificia Universidad Católica de Chile

Chile

\section{Enrique Sologuren Insúa \\ Universidad de los Andes \\ Chile}

\section{(c) $\underset{\mathrm{ar}}{\mathrm{a}} \mathrm{\textrm {N }}$}

María Natalia Castillo Fadić: Departamento de Ciencias del Lenguaje, Facultad de Letras, Pontificia Universidad Católica de Chile, Chile. I Correo electrónico: mcastilf@uc.cl

Enrique Sologuren Insúa: Instituto de Literatura, Facultad de Filosofía y Humanidades, Universidad de los Andes, Chile. | Correo electrónico: esologuren@miuandes.cl 


\section{Resumen}

Este artículo indaga en las desinencias del pretérito imperfecto de subjuntivo en el español de Chile, por medio de la descripción estadística de las realizaciones -ra y -se en el Corpus Básico del Español de Chile (Castillo Fadić, 2012). A través del análisis de distintas variables, con herramientas de lingüística computacional, lingüística de corpus y léxico-estadística, se busca precisar si estas desinencias, entendidas como variantes de una misma variable morfológica, alternan libremente o se correlacionan con otras variables. Los resultados del análisis muestran que existe correlación entre la variable morfológica en estudio y el mundo textual. Asimismo, se exploran factores gramaticales y léxico-semánticos que podrían influir en la elección de una u otra variante y que convendría estudiar en profundidad a partir de una muestra mayor.

Palabras clave: pretérito imperfecto de modo subjuntivo; alternancia de variantes -ra y -se; español de Chile; estadística léxica.

\section{Abstract}

This article studies desinences found in the imperfect subjunctive in Chilean Spanish, by means of the statistical description of -ra and -se verb endings that appear in the Corpus Básico del Español de Chile (Castillo Fadić, 2012). Through the analysis of different variables, and based on computational linguistics, corpus linguistics and lexical-statistical tools, we intend to define if these desinences, considered as variants of the same morphological variable, alternate freely or correlate with other variables. Results show the existence of a correlation between the morphological variable studied in the article and the textual world. In addition, grammatical and lexical-semantic factors that may influence on the choice of one or another variant are explored; it would be necessary to study these in depth considering a larger sample.

Keywords: The imperfect subjunctive; alternation of -ra and -se variants; Chilean Spanish variant; lexical statistics. 
A la memoria del Dr. Andrés Gallardo Ballacey

\section{Introducción}

El presente artículo se propone estudiar el pretérito imperfecto de modo subjuntivo en el español de Chile, que cuenta con dos posibles desinencias: -ra o -se. La bibliografía señala que ambas variantes alternan libremente, aunque algunos autores consideran que la variante -se está restringida a obras literarias o es preferente en este tipo de obras (cf., por ejemplo, RAE, 2010). La opción por una u otra variante parece obedecer a motivaciones dialectales, lo que justifica la necesidad de estudiar su comportamiento en el caso específico del español de Chile. Según la Nueva gramática de la lengua española, en el español de América se aceptan hoy las formas cantara y cantase en la lengua escrita, pero con una preferencia marcada por la primera: "Aun así, las formas en -se se usan ampliamente en América, en especial en la lengua literaria” (RAE, 2010: 1803).

La alternancia entre -se y -ra ya se encuentra documentada en los estudios señeros de Cuervo (1874 [1869]), quien estableció diferencias en la distribución de ambas formas en el español peninsular y en el español americano. Ahumada (1981) —desde un punto de vista dialectológico—- Wilson (1983) —desde una perspectiva diacrónica — y Gili Gaya —desde un enfoque dialectal y diafásico - constituyen otro cuerpo de investigaciones relevantes en torno a este problema; de sus postulados, destacamos la afirmación de este último autor (1983: 180), que estima que -ra es menos frecuente que -se en la conversación española, pero más frecuente en la escritura y entre hablantes más educados.

Años más tarde, DeMello (1993) investiga ambas variantes en ocho capitales americanas y en dos ciudades españolas - Madrid y Sevilla - y concluye que, aunque -ra y -se se mantienen, -se es considerablemente menos frecuente que -ra; agrega que desde la década de 1950 el uso de -se resulta poco natural e incluso propio de escritores pedantes (DeMello, 1993: 237) en distintas variedades de español, entre las que menciona como excepción la variedad chilena, junto con la argentina y la cubana. A nuestro juicio, el hecho de que el español de Chile parezca presentar un comportamiento de las variantes -ra y-se diferente del descrito para el español general da cuenta de la importancia de contar con estudios sintópicos específicos que enriquezcan, complementen y precisen el comportamiento de esta alternancia en la mencionada variedad.

Aunque los estudios de las variantes -ra y -se en imperfecto de subjuntivo fundamentados en lingüística de corpus y método cuantitativo son escasos, podemos mencionar los trabajos de Schwenter (2013), Elias y otros (2014) y Guzmán Naranjo (2016); todos ellos operan sobre corpus orales y coinciden en la necesidad de indagar en las propiedades estadísticas y de distribución de ambas variantes, así como en los factores que inciden en la elección de una u otra. Estimamos, por tanto, necesario continuar en esta línea, ahora sobre corpus escritos. 
Así, nuestra investigación se centra en las realizaciones -ra y -se en verbos modales del imperfecto de subjuntivo en el Corpus Básico del Español de Chile @ c. Se inscribe en el ámbito de la estadística léxica, la lexicología y la morfología, y emplea herramientas de lingüística de corpus y lingüística computacional. La hipótesis que sustentamos es que la opción por una u otra variante presenta correlación con variables lingüísticas y extralingüísticas. El estudio está centrado en el nivel morfológico, es de naturaleza empírica, finalidad pura e implicancias aplicadas, de corte no experimental, sincrónico-sintópico y presenta un alcance exploratorio-descriptivo.

Nuestro objetivo general es describir estadísticamente las realizaciones del imperfecto de subjuntivo en el español de Chile. Nuestros objetivos específicos son: a) identificar la distribución del pretérito imperfecto de subjuntivo por mundo, b) determinar la frecuencia relativa $\left(\mathrm{f}_{\mathrm{i}}\right)$ de las variantes -ra y -se por mundo, $\mathrm{c}$ ) precisar si la elección de una u otra variante se relaciona con el mundo en el que se presenta el verbo, d) determinar si la opción por una u otra variante tiene relación con el vocablo de que se trate y e) precisar si la opción por una u otra variante se relaciona con la persona y número de la desinencia verbal.

Este artículo se organiza del modo siguiente: para comenzar, se exponen los principales útiles conceptuales de relevancia para el análisis; luego, se presenta la metodología empleada en el estudio; por último, se plantean y discuten los principales resultados obtenidos; finalmente, se concluye con una síntesis de los principales hallazgos y se proyectan nuevos caminos para la investigación de aspectos morfosintácticos del español de Chile.

\section{El pretérito imperfecto de subjuntivo desde el punto de vista estadístico 2.1. Tiempo imperfecto, modo subjuntivo}

El modo subjuntivo español es considerado el más rico morfológicamente y el más complejo sintácticamente entre los de todas las lenguas romances - a excepción del portugués- (cf., por ejemplo, Fernández, 1990, y Dudemaine, 1998). Dentro del subjuntivo, el pretérito imperfecto es el tiempo más complejo, tanto por los contextos sintácticos en los que se usa como por la diversidad de los significados que expresa (cf. RAE, 2010). Además, presenta dos variantes en español: -ra y -se; la primera proviene del pluscuamperfecto del indicativo latino, y la segunda, del pluscuamperfecto de subjuntivo de la misma lengua.

En el español europeo, la alternancia entre estas dos formas es prácticamente libre; tanto en la escritura como en la oralidad predomina la variante -ra, aunque en menor medida que en América. Al comparar la modalidad escrita con la oral, se observa que en la primera aumenta la proporción de -se, pese a que sigue siendo minoritaria respecto de -ra. En el español americano, en tanto, "las variantes en -se se consideran demasiado elevadas, o incluso afectadas, en la lengua oral de algunos países americanos" (RAE, 2010: 1803). 
En relación con los contextos sintácticos que excluyen una de las formas en favor de otra, estos son muy escasos. Uno de estos corresponde al uso de los verbos modales querer, podery deber, donde, según RAE (2010: 1804), -ra no alterna con-se, pero sí con el condicional; esta alternancia sería general en los verbos modales poder y deber, pero no en las prótasis condicionales (debieras-deberías esforzarte más); el auxiliar querer, por su parte, se suma, especialmente en fórmulas de cortesía como en los casos 'quisiera-querría pedirle un favor' (RAE, 2010: 1807).

Respecto de las variables que podrían explicar la alternancia, Schwenter (2013: 7) señala que la persona y el número tienen algún tipo de efecto en la elección de una forma determinada, pero no provee datos concretos que respalden esta afirmación. En su explicación también recurre a variables de tipo individual, como el efecto de priming o cebado de tipo cognitivo (Lundh y Czyzykow-Czarnocka, 2001; Schwenter, 2013), según el cual cuando el orador utiliza la forma -se es más probable que la utilice nuevamente cuando requiera producir una construcción en imperfecto del subjuntivo.

También desde otros enfoques, como la teoría de la construcción de la morfología (CM) (Booij, 2010), se ha intentado explicar el fenómeno de la alternancia -ra/-se en español. En esta línea, destaca el trabajo de Guzmán Naranjo (2016): para este autor, tanto la explicación que asume que -se y -ra son alomorfos que varían libremente como aquella que los conceptualiza como morfemas diferentes (Asratian, 2007; Lloret, 2011; RAE, 2010) son problemáticas, ya que no son capaces de explicar ni las diferencias sistemáticas ni las similitudes e identidad en la función gramatical. Por ello, propone una alternativa intermedia — "In this analysis both -se and -ra are more specific constructions that instantiate the more general abstract construction” (Guzmán Naranjo, 2016: 5)—, según la cual ambas formas comparten la misma función gramatical, aunque presentan importantes diferencias, lo que se sustenta en la hipótesis de que la elección de una u otra forma dependerá parcialmente de otras variables lingüísticas — no exclusivamente gramaticales_y no lingüísticas — fundamentalmente pragmáticas-.

Guzmán Naranjo (2016: 31) reporta que, en el marco de su estudio basado en el Corpus Oral de Referencia de la Lengua Española Contemporánea(CORLEC),-ra es considerablemente más frecuente que -se en el español hablado, lo que concuerda con los resultados obtenidos por DeMello (1993) y Rojo (2008). Asimismo, sus datos parecen indicar correlaciones débiles con factores gramaticales de tipo morfológico, pero efectos fuertes de tipo léxico, con una marcada preferencia de -ra por los verbos modales. En ese sentido, -ra atraería verbos modales, mientras -se tendería a repelerlos.

\subsection{La estadística léxica}

La estadística léxica o léxico-estadística forma parte de la lingüística cuantitativa. Supera la noción de palabra y opera a partir de las nociones de vocablo y tipo. Si la palabra es la unidad 
mínima textual, el vocablo es la unidad mínima lexicológica (cf. Müller, 1973: 225-226), que puede identificarse con el lema o unidad de cita, variable o invariante, mejor representante de un paradigma de variantes que comparten una base común; el tipo es una unidad de moderada abstracción, que permite agrupar y contabilizar las unidades léxicas que comparten significado y significante y que se agrupan bajo un vocablo en calidad de variantes (cf. Lara, 2006: 138; y Castillo Fadić, 2015b: 2). La palabra, en tanto, corresponde al nivel del texto. Por ejemplo, en la oración "El hombre hablaba y hablaba y no paraba de hablar" tenemos diez palabras, ocho tipos y sólo siete vocablos; si nos centramos en el vocablo "hablar", vemos que presenta dos tipos - "hablar" y "hablaba"-, los que en el texto se actualizan por medio de tres palabras.

\section{Metodología}

\subsection{Obtención y procesamiento del corpus}

A partir del Corpus Básico del Español de Chile (Castillo Fadić, 2012), corpus de referencia etiquetado de más de 500.000 palabras en contexto distribuidas en cinco mundos -Drama, Narrativa, Ensayo, Técnico-Científico y Prensa-, se realizó una búsqueda por tipos marcados con las etiquetas EAGLES ${ }^{1}$ VMSIISO, VMSIIPO, VMSI2SO, VMSI2Po y VMSI3SO, correspondientes a verbos modales - VM - de modo subjuntivo - S - y tiempo imperfecto - I-, de primera persona singular y plural — 150 y $\mathrm{IPO}$-, segunda persona singular y plural $-2 \mathrm{So}$ y $2 \mathrm{Po}-$ y tercera persona singular y plural —3So y 3Po-. Esta búsqueda permitió no sólo identificar todas las unidades léxicas verbales conjugadas en imperfecto de subjuntivo, sino también apreciar su distribución en términos de la frecuencia absoluta $\left(\mathrm{F}_{\mathrm{i}}\right)$ por mundo y total, dispersión (D) y uso (U). Cabe precisar que para efectos de segmentación las locuciones verbales fueron tratadas como verbos.

\subsection{Descripción y análisis}

El corpus quedó constituido por 500 tipos, correspondientes a 359 vocablos. De ellos, 235 presentaron $F_{i}=1$ y, por ende, $D=0$ y $U=0$. Los datos se trasladaron a una hoja de cálculo y se revisó la $F_{i}$ y $f_{i}$ de tipos y vocablos por mundo, la distribución de las variantes -ra y -se por mundo -incluyendo las realizaciones voseantes-, y su distribución por vocablo y por persona y número.

$1 \quad$ Esta búsqueda fue posible gracias al procesamiento realizado con LexBas 1.0, producto basado en Freeling 2.2 y creado ad hoc, gracias al financiamiento y patrocinio del Dr. Humberto López Morales, para la obtención del Léxico Básico del Español de Chile a partir de nuestro corpus (cf. Castillo Fadić, 2012 y Castillo Fadić, 2015a). 


\subsection{Test estadísticos utilizados}

Se aplicó un conjunto de pruebas estadísticas para determinar si la co-variación entre las variables independientes - mundo, vocablo, persona y número-y la variable dependiente - la desinencia del pretérito imperfecto de subjuntivo, con sus variantes -ra y -se- era estadísticamente significativa.

3.3.1. ANOVA de un factor: mediante esta técnica estadística, se evalúa la relación entre una variable independiente y una variable dependiente; aunque constituye una extensión de la tStudent, su ventaja para efectos de esta investigación es que permite comparar medias de tres o más grupos, lo que posibilita observar el comportamiento de nuestra variable dependiente - la desinencia del pretérito imperfecto subjuntivo - en relación con cada variable independiente considerada, tanto por separado como de manera combinada. Dado que el total de ocurrencias de las piezas morfológicas -ra y -se es distinto en cada mundo y presenta también diferente $F_{i}$ por vocablo, persona y número, se aplicaron además dos pruebas post hoc, cuyo uso está estrechamente relacionado con el análisis de la varianza (ANOVA). Estas son las siguientes:

3.3.2. Scheffé: se trata de un test de comparaciones múltiples, que coteja todos los posibles pares de medias y utiliza como error típico el valor de la varianza residual obtenido mediante el test ANOVA: "The Scheffé test is the most conservative of all the possible post hoc tests. It controls Type I error for doing all possible post hoc comparisons, not just pair-wise mean comparisons" (Pagano, 2012: 425). Así, esta prueba permite corregir el error proveniente de la aplicación de la ANOVA.

3.3.3. Kruskall-Wallis: esta prueba post hoc no asume la normalidad de los datos: "The Kruskal-Wallis test does not assume population normality or homogeneity of variance, as does parametric ANOVA, and requires only ordinal scaling of the dependent variable" (Pagano, 2012: 507). En este sentido, su uso se justifica de manera complementaria cuando existen diferencias importantes en el total de ocurrencias del imperfecto del subjuntivo en cada uno de los mundos y, por lo tanto, la homogeneidad de la varianza es extrema.

Los resultados obtenidos luego de la aplicación de cada una de las pruebas estadísticas serán desarrollados en la sección siguiente.

\section{Resultados y discusión}

\subsection{Verbos modales: imperfecto de subjuntivo por mundo}

En lo que concierne a los verbos modales o principales, el uso del imperfecto de subjuntivo se da preferentemente en Narrativa (37\%) y Drama (24\%) y resulta menos frecuente en Ensayo (15\%), Técnico-Científico (12\%) y Prensa (12\%). 


\subsection{Desinencias -ra y -se por mundo}

La desinencia -ra es preferente y alcanza el 90\% de las realizaciones, si consideramos su $\mathrm{F}_{\mathrm{i}}$ total; -se, en tanto, sólo llega al 10\%.

Si observamos la distribución por mundos (cf. figura 1), se aprecia una mayor $F_{i}$ del subjuntivo imperfecto en Narrativa, seguido por Drama. Ensayo, Técnico-Científico y Prensa, presentan las menores $F_{i}$ de este tiempo y modo. Esto podría explicar la percepción de que la variante -se es preferente en Narrativa. Podemos afirmar que no es que esta variante tenga mayor $f_{i}$ en Narrativa, sino que es el imperfecto subjuntivo el que presenta mayor $F_{i}$ en Narrativa, lo que da mayores posibilidades de aparición a ambas variantes.

\section{FIGURA 1}

Distribución de $F_{i}$ de imperfecto subjuntivo por mundo: desinencias

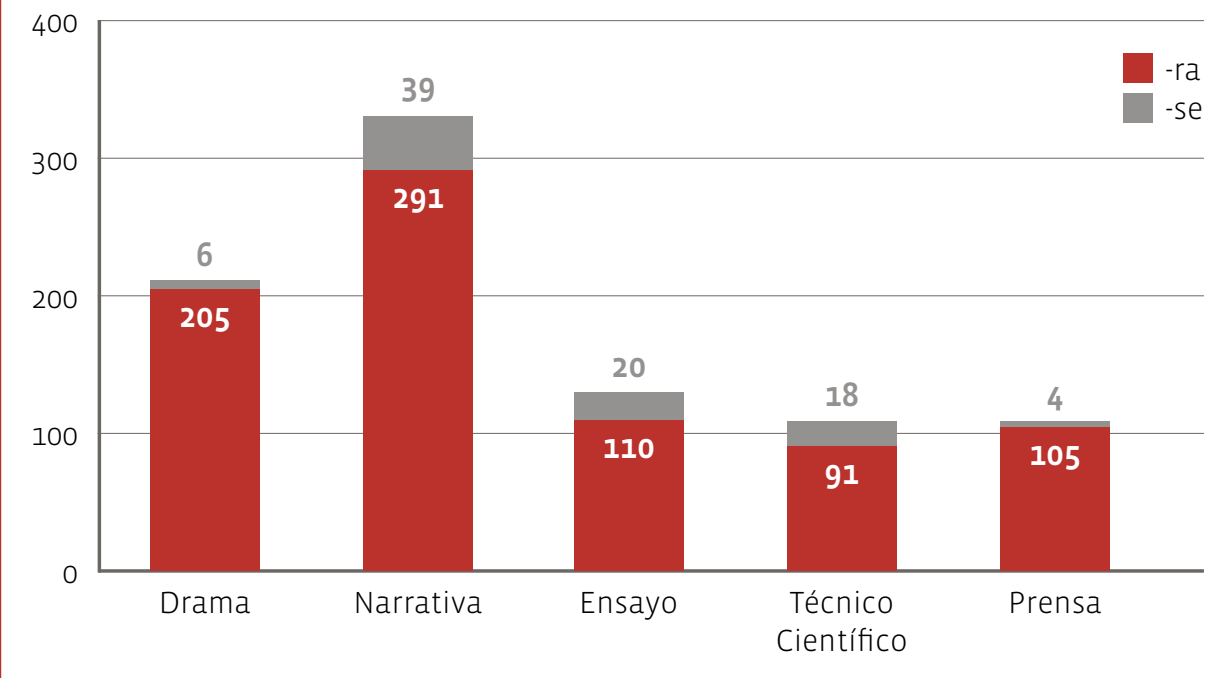

Sin embargo, -ra y -se no se distribuyen equitativamente por mundo, ni respecto del total de la muestra, ni respecto de las realizaciones de subjuntivo imperfecto estratificadas por mundos. De este modo, podemos observar una correlación entre cada desinencia y el mundo preferente de aparición.

Como se aprecia en la figura 2, el porcentaje de subjuntivo por mundo no es en ningún caso proporcional al de la preferencia por una desinencia u otra. Así, Drama, el segundo mundo en usar el subjuntivo imperfecto, presenta la más baja $f_{i}$ de la variante -se. Técnico-Científico, por su parte, el mundo con la $F_{i}$ más baja de pretérito imperfecto subjuntivo junto con Prensa, resulta ser el que tiene la mayor $f_{i}$ de-se, seguido por Ensayo, el mundo con la segunda $F_{i}$ más baja de pretérito imperfecto subjuntivo. 


\section{FIGURA 2}

Frecuencias relativas de desinencias en cada mundo: síntesis

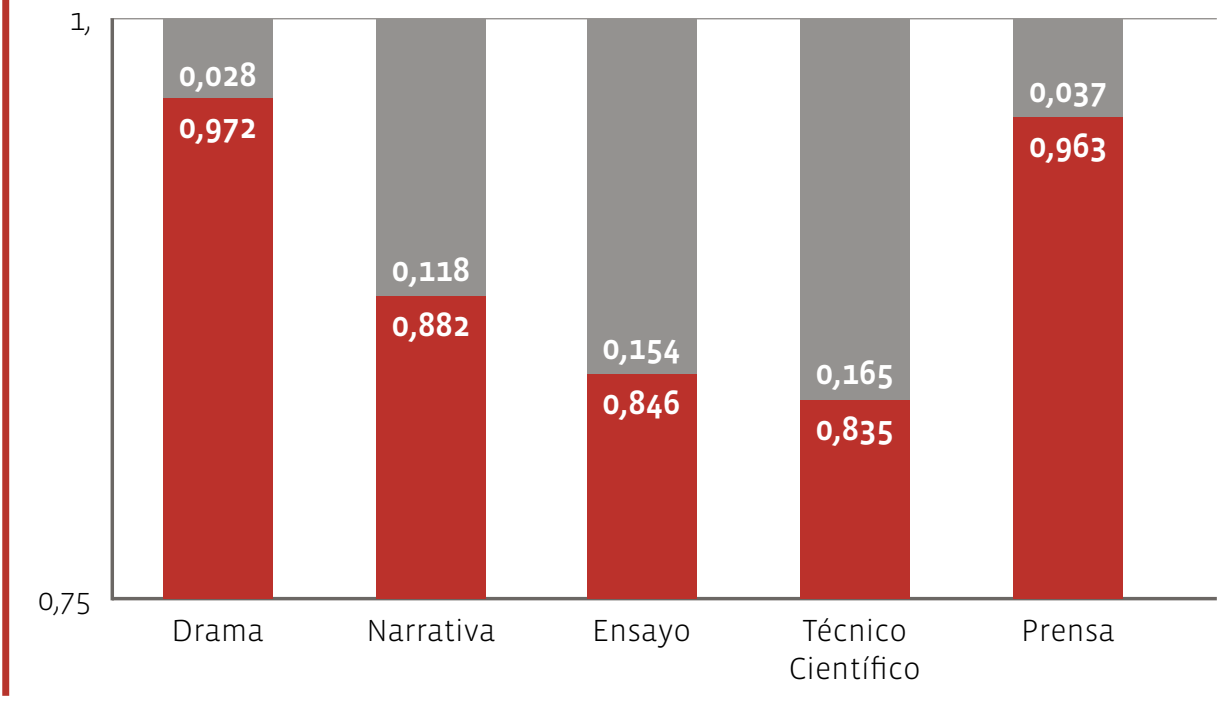

Drama (97\%) y Prensa (96\%) resultan comparables en la amplia supremacía de la desinencia -ra sobre-se (3\% y $4 \%$ respectivamente), aunque Drama duplica a Prensa en la $F_{i}$ de imperfecto subjuntivo (cf. figura 1 ).

Narrativa, el mundo de más recurrente aparición de imperfecto subjuntivo, prefiere la desinencia en -ra en un $88 \%$ de los casos y emplea la variante -se sólo en un $12 \%$. Una distribución similar se observa en Ensayo (84\% y 16\%) y Técnico-Científico (83\% y 17\%), los mundos con mayor $f_{i}$ de la variante -se.

Se realizó luego una serie de pruebas de comparación de medias (Pagano, 2012). Primero, un ANOVA de un factor, que mostró una correlación significativa entre el mundo y la distribución de la desinencia, con un p-valor menor a 0,05, como se observa en la figura 3.

Ya que el total de las ocurrencias del imperfecto subjuntivo es distinto en cada mundo, se aplicaron dos pruebas post hoc: Scheffé y Kruskall-Wallis; ambas buscan correlaciones entre la distribución de las desinencias, comparando cada mundo con otro, par por par. Los resultados de ambas mostraron que al comparar las medias por mundo, estos se agrupan en dos conjuntos: el primero, compuesto por Narrativa, Ensayo y Técnico-Científico, con una media significativamente menor en la frecuencia de -ra, y el segundo, formado por Prensa y Drama (cf. figura 4). Esto da cuenta de que la variante -ra es significativamente más frecuente en Drama y en Prensa, aunque el $n$ sea distinto.

Ambos conjuntos pueden ilustrarse más claramente en un gráfico de medias (cf. figura 5). 


\section{FIGURA 3}

ANOVA para mundo

\section{DESCRIPTIVOS}

Mundo

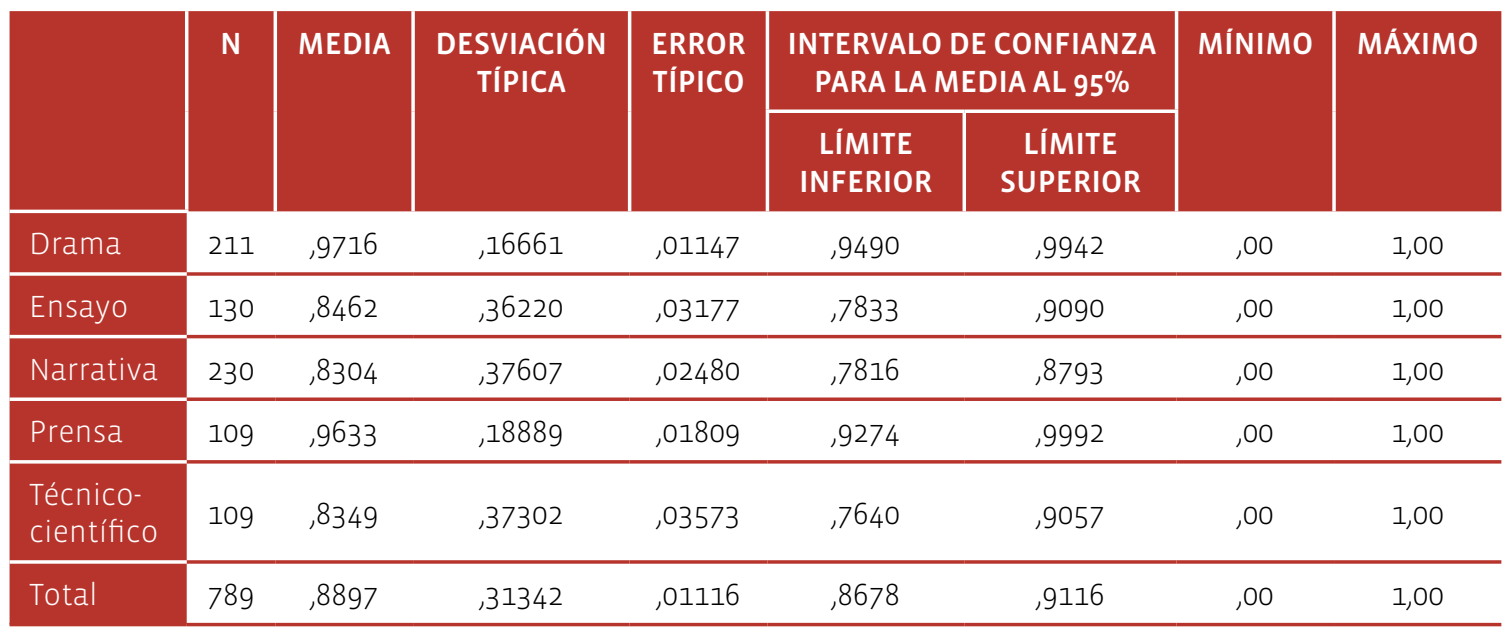

PRUEBA DE HOMOGENEIDAD DE VARIANZAS

Mundo

\begin{tabular}{c|c|c|c} 
ESTADISTICO DE LEVENE & GL1 & GL2 & SIG. \\
\hline 45,764 & 4 & 784 &, 000 \\
\hline
\end{tabular}

ANOVA DE UN FACTOR

Mundo

\begin{tabular}{l|c|c|c|c|c} 
& $\begin{array}{c}\text { SUMA DE } \\
\text { CUADRADOS }\end{array}$ & GL & $\begin{array}{c}\text { MEDIA } \\
\text { CUADRÁTICA }\end{array}$ & F & SIG. \\
\hline Inter-grupos & 3,387 & 4 &, 847 & 8,968 &, 000 \\
\hline Intra-grupos & 74,020 & 784 &, 094 & & \\
\hline Total & 77,407 & 788 & & \\
\hline
\end{tabular}

\subsubsection{Voseo morfológico en imperfecto de subjuntivo}

El corpus presenta cinco ocurrencias de voseo morfológico en la modalidad chilena, todas ellas en -ra. Los cinco casos tienen lugar en Drama ( $D=0$ y $U=0)$ y corresponden a realizaciones

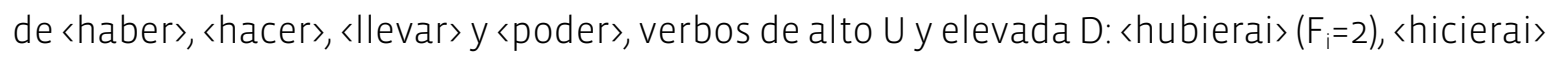
$\left(F_{i}=1\right)$, <llevarai $\left(F_{i}=1\right)$ y $\langle$ pudierai $\rangle\left(F_{i}=1\right)$. 


\section{FIGURA 4}

Test de Scheffé: mundo, distribución y valor $p$
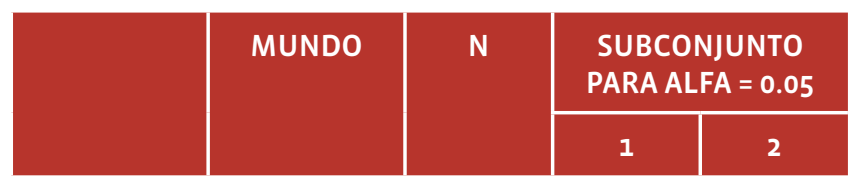

\begin{tabular}{lllll} 
& Narrativa & 230 &, 8304 & \\
\cline { 2 - 5 } Schefféa,b $^{\text {Técnico }}$ & 109 &, 8349 & \\
\cline { 2 - 5 } & Ensayo & 130 &, 8462 & \\
\cline { 2 - 5 } & Prensa & 109 & &, 9633 \\
\cline { 2 - 5 } & Drama & 211 & &, 9716 \\
\hline & Sig. & &, 996 & 1,000 \\
\hline
\end{tabular}

\section{FIGURA 5}

Test de Scheffé: gráfico de medias

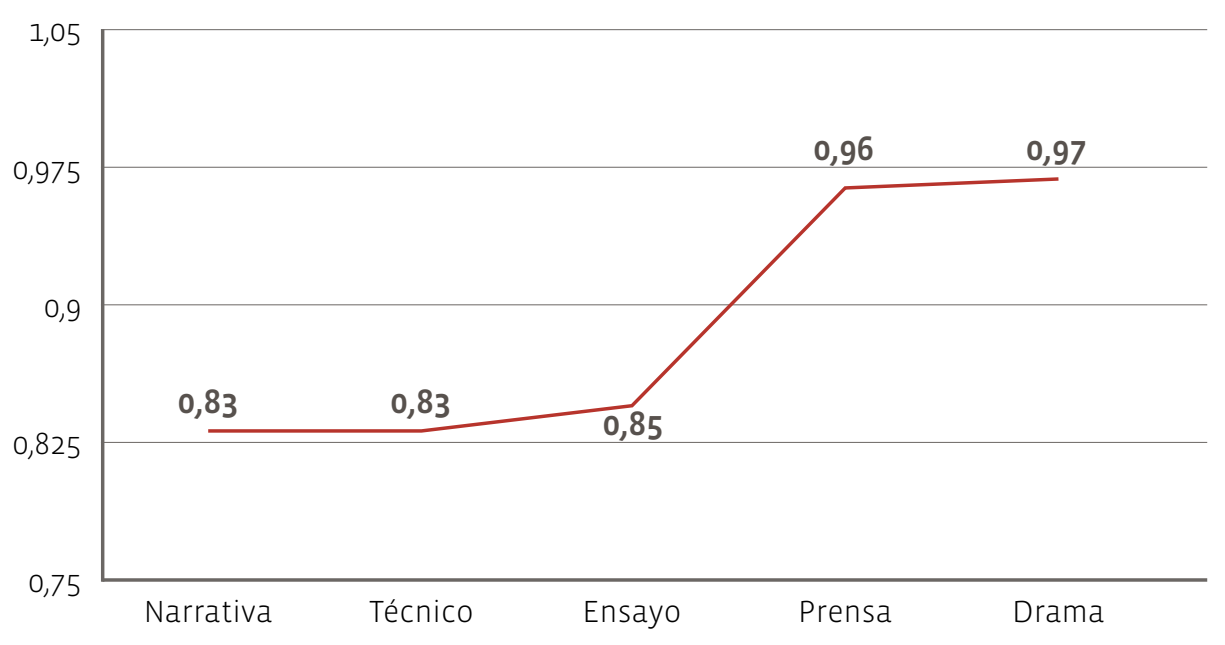

\subsection{Desinencias en -ra y en -se por vocablo}

Como señalamos en 3.2, el corpus quedó constituido por 359 vocablos, de los cuales 235 presentaron $\mathrm{F}_{\mathrm{i}}=1 \mathrm{y}$, por ende, $\mathrm{D}=0$ y $\mathrm{U}=0$. De estos, sólo 21 se realizaron exclusivamente en -se. Estos son:

$$
\begin{aligned}
& \text { - abandonar } \\
& \text { - apoderar } \\
& \text { - atentar }
\end{aligned}
$$
- confeccionar
- contemplar
- demorar
- descartar
- descerrajar
- empuñar


- formar_parte

- hundir

- ignorar

- notar
- presagiar

- procurar

- reanudar

- rebelar
- resbalar

- sacudir

- sentar

- suceder

Los demás, en tanto, sólo presentaron la variante -ra. Sólo uno de los verbos que presentó exclusivamente la variante -se tuvo $\left.F_{i}\right\rangle$ : se trata de 〈solicitar $\rangle$, con $F_{i}=2$.

Seis verbos se realizaron indistintamente por medio de las variantes -ra o-se, con idéntica $f_{i}$. Es el caso de:

- declarar
- garantizar

\author{
- perpetuar \\ - poseer
}

- prestar

- resultar

Por último, 28 verbos presentaron ambas variantes, con distintas $f_{i}$, como se ilustra en la figura 6. En todos los casos, la variante-ra fue preferente.

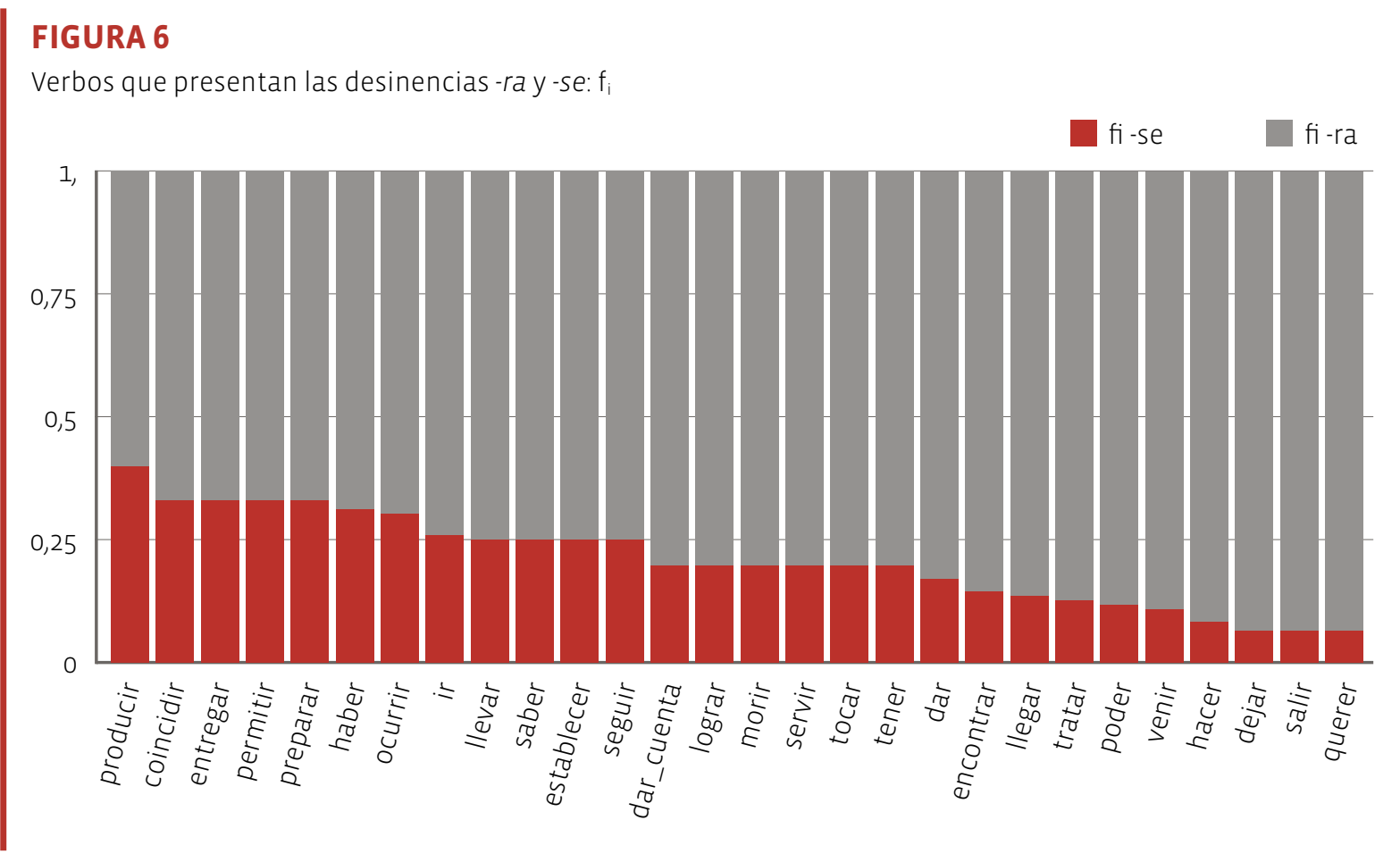

Respecto de los tres verbos que RAE (2010) menciona como los que pueden ver aumentada su frecuencia de -ra porque en esa variante pueden alternar con condicional, sólo 〈poder > se emplea exclusivamente en -ra y, en efecto, tiende a usarse en alternancia con el condicional, en casos como "La voluntad del difunto, pudiera inducir a un desenlace diferente" 
(Drama), “[...] cuando dejái de tomar, parece que todavía pudierai servir para algo” (Drama) o "[...] sacando fuerzas de flaqueza, de la que [...] bien pudiera ser la antesala de su declive terminal” (Ensayo).

Aunque los resultados de esta exploración resultan interesantes, el reducido tamaño del $n$ de cada vocablo dificulta el análisis. Por ello, para las pruebas estadísticas se consideraron sólo los vocablos con un $n \geq 10$. Se aplicaron los mismos tests (ANOVA de un factor, Scheffé y Kruskall-Wallis) y ninguno fue significativo. Si revisamos, por ejemplo, la figura 7, podemos notar que el p-valor es mayor a 0,05, por lo que las correlaciones entre vocablo y desinencia no son estadísticamente significativas.

\section{FIGURA 7}

ANOVA para vocablo

\section{DESCRIPTIVOS}

Vocablos

\begin{tabular}{|c|c|c|c|c|c|c|c|c|}
\hline & $\mathbf{N}$ & MEDIA & $\begin{array}{l}\text { DESVIACIÓN } \\
\text { TÍPICA }\end{array}$ & $\begin{array}{l}\text { ERROR } \\
\text { TIPICO }\end{array}$ & $\begin{array}{r}\text { INTERVALO } \\
\text { PARA LA } ~\end{array}$ & $\begin{array}{l}\text { CONFIANZA } \\
\text { DIA AL } 95 \%\end{array}$ & MÍNIMO & MÁXIMO \\
\hline & & & & & $\begin{array}{l}\text { LÍMITE } \\
\text { INFERIOR }\end{array}$ & $\begin{array}{c}\text { LIIMITE } \\
\text { SUPERIOR }\end{array}$ & & \\
\hline dar & 12 & 8333 & ,38925 & 11237 &, 5860 & 1,0807 &, 00 & 1,00 \\
\hline dejar & 15 & ,9333 & ,25820 & ,06667 & ,7903 & 1,0763 & ,00 & 1,00 \\
\hline haber & 19 &, 6842 & ,47757 & 10956, &, 4540 &, 9144 &, 00 & 1,00 \\
\hline hacer & 24 & 9167 & ,28233 & ,05763 & 7974 & 1,0359 &, 00 & 1,00 \\
\hline ir & 19 &, 7368 & ,45241 & 10379, &, 5188 & 9549 & , 00 & 1,00 \\
\hline llegar & 15 & 8667 & 35187 & ,09085 & 6718 & 1,0615 & ,00 & 1,00 \\
\hline ocurrir & 10 & ,7000 &, 48305 & 15275 &, 3544 & 1,0456 & , 00 & 1,00 \\
\hline poder & 67 & ,8806 & 32671 & 03991 & 8009 &, 9603 &, 00 & 1,00 \\
\hline querer & 31 & 9355 & 24973 & ,04485 & ,8439 & 1,0271 & , 00 & 1,00 \\
\hline salir & 15 & ,9333 & ,25820 & ,06667 & ,7903 & 1,0763 &, 00 & 1,00 \\
\hline tener & 31 &, 8065 & 40161 & ,07213 & ,6591 &, 9538 &, 00 & 1,00 \\
\hline Total & 258 & 8527 & 35508 & 02211 & 8092 & 8962 & ,00 & 1,00 \\
\hline
\end{tabular}

PRUEBA DE HOMOGENEIDAD DE VARIANZAS

Vocablos

\section{ESTADÍSTICO DE LEVENE} 4,987

\section{GL1}

GL2

247
SIG.

, 000 


\begin{tabular}{|c|c|c|c|c|c|}
\hline \multicolumn{6}{|c|}{ ANOVA DE UN FACTOR } \\
\hline & $\begin{array}{c}\text { SUMA DE } \\
\text { CUADRADOS }\end{array}$ & GL & $\begin{array}{c}\text { MEDIA } \\
\text { CUADRATICA }\end{array}$ & $\mathbf{F}$ & SIG. \\
\hline Inter-grupos & 1,659 & 10 & 166 & 1,333 & 213 \\
\hline Intra-grupos & 30,744 & 247 & ,124 & & \\
\hline Total & 32,403 & 257 & & & \\
\hline
\end{tabular}

\subsection{Desinencias en -ra y en -se por persona y número}

Así como el imperfecto de subjuntivo se distribuye desigualmente en los distintos mundos, su distribución resulta también desigual en lo que concierne a persona y número, como se aprecia en las figuras 6 y 7. Y aunque podemos concluir fácilmente que la primera persona singular es la que presenta una mayor $F_{i}$ de imperfecto subjuntivo en todos los mundos, por separado y en total, no es posible extrapolar esa conclusión a una pretendida mayor fi de la variante -se en esa persona y número. En efecto, siendo la $F_{i}$ de imperfecto subjuntivo mayor en primera persona singular que en las demás, hay en esa persona y número mayor posibilidad de aparición de ambas desinencias, lo que explica que, en términos de $F_{i}$, la variante -se tenga el mayor número de ocurrencias en primera persona singular (cf. figura 8).

\section{FIGURA 8}

Imperfecto de subjuntivo por persona y número en cada mundo $\left(\mathrm{F}_{\mathrm{i}}\right)$

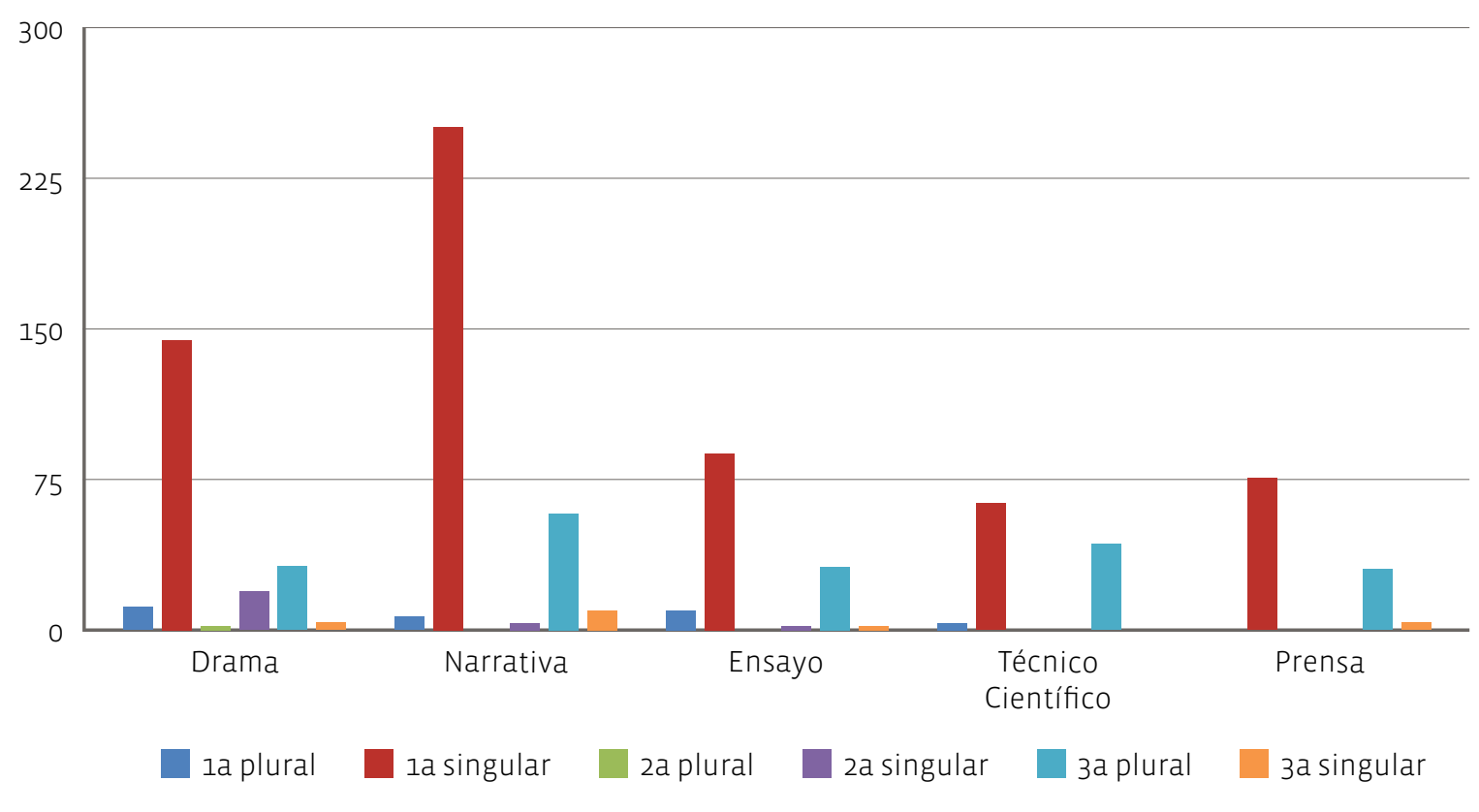




\section{FIGURA 9}

Distribución imperfecto subjuntivo y de desinencias por persona y número $\left(F_{i}\right)$

\begin{tabular}{l|c|c|c|c|c|c} 
& $\begin{array}{c}\mathbf{1}^{\text {a }} \\
\text { SINERS. }\end{array}$ & $\begin{array}{c}\mathbf{2}^{\text {a }} \text { PERS. } \\
\text { SING. }\end{array}$ & $\begin{array}{c}\mathbf{3}^{\text {a }} \text { PERS. } \\
\text { SING. }\end{array}$ & $\mathbf{1}^{\text {a PERS. PL. }}$ & $\mathbf{2}^{\text {a PERS. PL. }}$ & $\mathbf{3}^{\text {a PERS. PL. }}$ \\
\hline -RA & 567 & 18 & 12 & 28 & 1 & 171 \\
\hline -SE & 56 & 0 & 6 & 3 & 0 & 0 \\
\hline -RAI (VOSEO) & 0 & 5 & 0 & 0 & 31 & 1 \\
\hline TOTAL & 623 & 23 & 18 & 31 & 0 \\
\hline
\end{tabular}

Y aunque la tercera persona singular parece ser la que más favorece la aparición de la variante -se, que alcanza un 33\% de las realizaciones (cf. figura 10), lo cierto es que el reducido tamaño de las muestras por persona y número dificulta realizar un análisis estadístico; en el caso específico de la tercera persona singular, esta presenta la segunda $F_{i}$ más baja de imperfecto subjuntivo, después de la segunda persona de plural (cf. figura 9). La segunda persona singular y la plural, en tanto, sólo se realizan por medio de la variante -ra.

\section{FIGURA 10}

Distribución de desinencias por persona y número

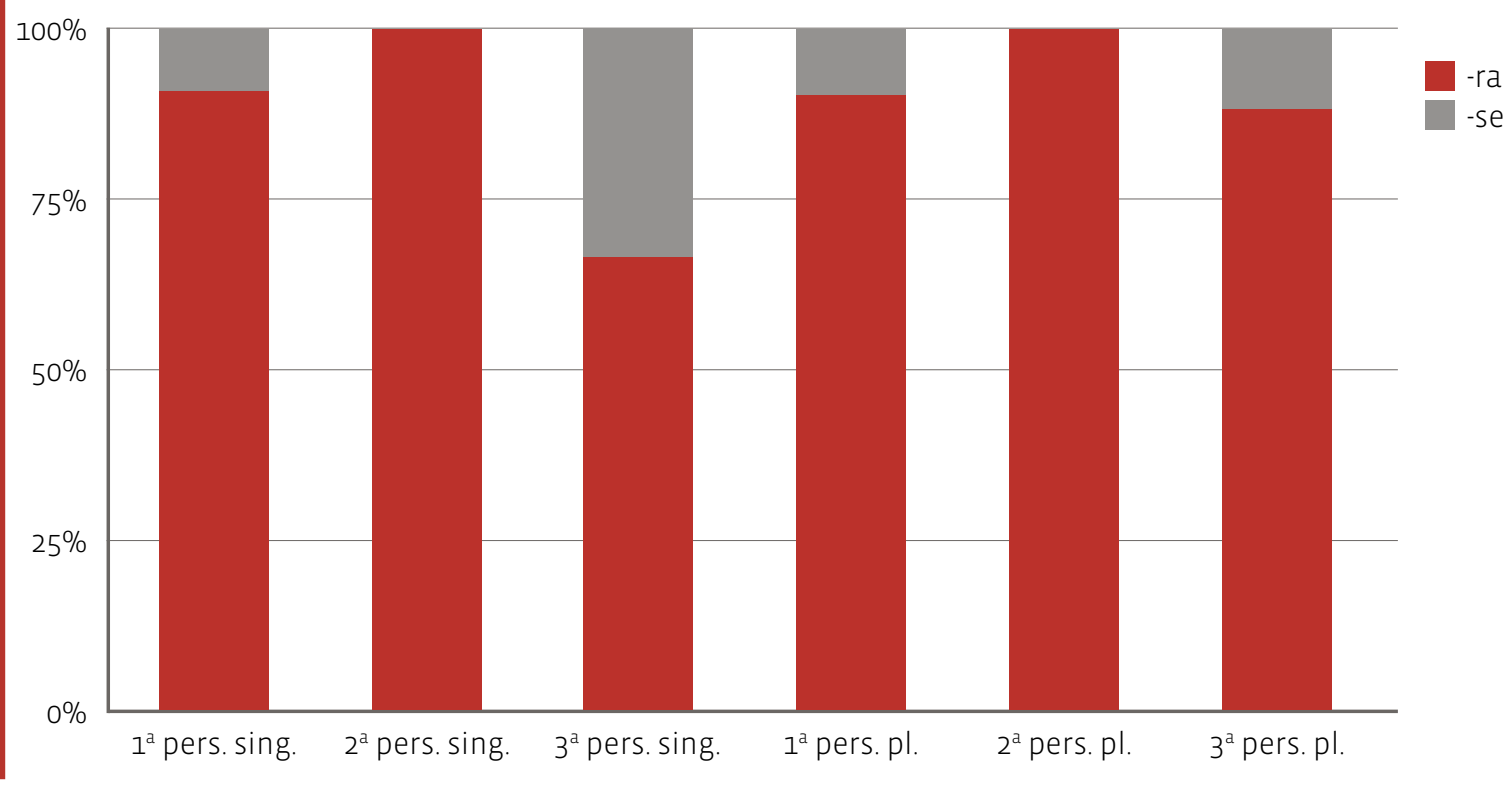

La primera persona singular y plural y la tercera persona plural presentan respectivamente un $9 \%$, un $10 \%$ y un $11 \%$ de ocurrencias para la variante -se, como se ilustra en la figura 10. 
Dado el reducido tamaño de la muestra en lo relativo a persona y número, para las pruebas estadísticas se usaron sólo tres conjuntos: $1^{a}$ persona singular, $3^{a}$ persona plural y "otros", categoría en la que se colapsaron todas las demás. Se realizaron las mismas tres pruebas (ANOVA de un factor, Scheffé y Kruskall-Wallis) y los resultados no fueron significativos.

\section{FIGURA 11}

ANOVA para persona y número

\section{DESCRIPTIVOS}

Persona y número

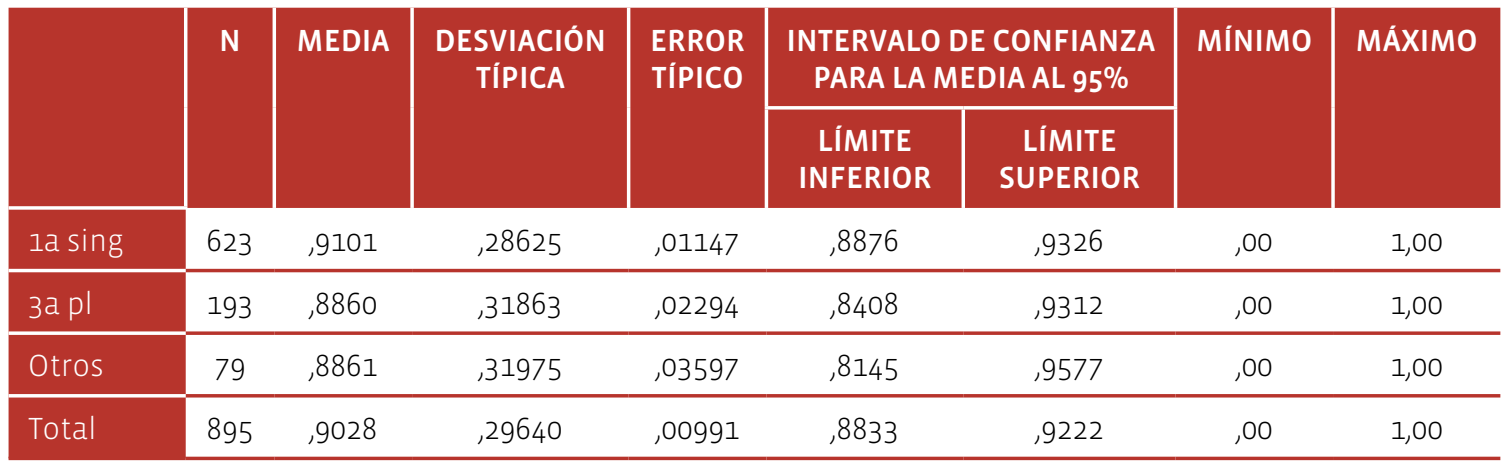

PRUEBA DE HOMOGENEIDAD DE VARIANZAS

Persona y número

\begin{tabular}{r|c|c|c}
\hline ESTADISTICO DE LEVENE & GL1 & GL2 & SIG. \\
\hline 2,451 & 2 & 892 &, 087 \\
\hline
\end{tabular}

\section{ANOVA DE UN FACTOR}

Persona y número

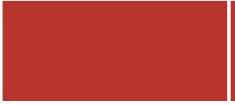

SUMA DE CUADRADOS

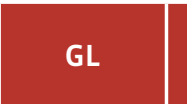

\section{MEDIA CUADRÁTICA}

Inter-grupos , 110

2

, 055

Intra-grupos

$78,433 \quad 892$
,088

$\begin{array}{lll}\text { Total } & 78,543 & 894\end{array}$

En la figura 11, se detallan las tablas de coeficientes de la prueba estadística ANOVA. Estos descriptivos dejan evidencia de que, con los datos actuales, no es posible determinar significancia estadística entre persona, número y desinencia. En ese sentido se requieren nuevos estudios en diferentes corpus del español. 


\section{Conclusiones}

1. Existe una correlación significativa entre tiempo, modo y mundo. El imperfecto de subjuntivo es preferente en Narrativa (37\%) y Drama (24\%) y resulta menos frecuente en Ensayo (15\%), Técnico-Científico (12\%) y Prensa (12\%).

2. Aunque en todos los mundos la variante -ra es preferente, existe una correlación significativa entre la variable morfológica estudiada y la variable mundo. Así, la fi de -ra es significativamente superior en el conjunto de Drama y Prensa que en el de Narrativa, Ensayo y Técnico-Científico.

3. El voseo verbal se realiza únicamente en -ra y sólo se registra en Drama, el mundo más cercano a la oralidad. No obstante, el reducido $n$ no permite establecer una correlación significativa entre voseo verbal y mundo, ni entre voseo verbal y desinencia.

4. Algunos vocablos parecen tender más a una variante que a otra. No obstante, con los datos de que disponemos no es posible establecer una correlación significativa entre vocablos y variable morfológica. La gran mayoría de los verbos en imperfecto subjuntivo presenta $F_{i}=1$. De ellos, la mayoría sólo se realiza en -ra $\left(f_{i}=0,654\right)$, y la minoría, sólo en -se $\left(f_{i}=0,058\right)$. Los demás presentan ambas variantes, ya sea con idéntica $F_{i}\left(f_{i}=0,016\right)$ o con diferente $F_{i}\left(f_{i}=0,080\right)$. Esto podría obedecer a alguna variable escondida -área temática o ámbito disciplinar específico, por ejemplo— o a particularidades semánticas o gramaticales de los verbos que, eventualmente, podrían organizarse en clases. Incluso, podría haber una explicación diacrónica.

5. Aunque en todas las personas es preferente la variante -ra, en tercera persona singular la variante -se alcanza un tercio de las realizaciones, siendo la persona con mayor $f_{i}$ de -se. Sin embargo, el tamaño de la muestra por persona y número no permite afirmar que exista una correlación significativa entre persona, número y desinencia.

6. En una investigación futura, sería de interés estudiar por qué el $n$ varía tanto según persona y número y, especialmente, por qué el $n$ es tan superior en $1^{a}$ persona singular. Sería también relevante revisar todos los casos en los que el imperfecto de subjuntivo alterna con condicional, para determinar si, en el español de Chile, se trata de un fenómeno que afecta a los verbos mencionados por la RAE (2010) - querer, poder y deber-de manera exclusiva o más frecuente o preferente, o si se da también en otros verbos con similar $\mathrm{f}_{\mathrm{i}}$. Resultaría también interesante verificar si esta alternancia presenta correlación con otras variables, así como profundizar en las asociaciones que cada morfema establece con diferentes tipos de discurso. Por último, nos parece importante retomar la variable vocablo a partir de un $n$ mayor que contemple más vocablos y una muestra mayor de cada uno; esto permitiría, por ejemplo, analizar los verbos que parecen realizarse sólo en -se, para determinar si esta tendencia se mantiene en una muestra mayor y, de ser así, estudiar a qué obedece la opción exclusiva por una variante minoritaria en todos los demás casos. 
7. Esta investigación se ha centrado en los verbos modales; en un estudio próximo, pretendemos abordar los verbos auxiliares y semiauxiliares.

\section{Bibliografía citada}

Ahumada, Ignacio, 1981: Notas a la gramática de la lengua castellana de don Andrés bello, Bogotá: Instituto Caro y Cuervo.

Asratian, Arucia, 2007: “Variación -Ra/-Se en el Español Hablado en Caracas”, Boletín de Lingüística 19 (27), 5-41.

Boolı, Geert, 2010: “Construction morphology”, Language and Linguistics Compass 4 (7), 543-555.

Castillo Fadić, María Natalia, 2012: Corpus Básico del Español de Chile.

Castillo Fadić, María Natalia, 2015a: "Léxico Básico del Español de Chile: el proyecto", E-Aesla (1), 1-8 [http://cvc.cervantes.es/lengua/eaesla/pdf/01/51.pdf, fecha de consulta: 8 de agosto de 2016].

Castillo Fadić, María Natalia, 2015b: "El verbo <hacer> en el español de chile: tipos y combinaciones frecuentes en el género ensayo", E-Aes/a (1), 1-9 [http://cvc.cervantes.es/lengua/eaesla/ pdf/01/50.pdf, fecha de consulta: 8 de diciembre de 2016].

Cuervo, Rufino José, 1874 [1869]: "Observaciones sobre el Diccionario de la Real Academia Española” en Disquisiciones sobre filología castellana, 119-145.

DeMello, George, 1993: “-ra vs.-se subjunctive: A new look at an old topic”, Hispania 76 (2), 235-244.

Dudemaine, Céline, 1998: “Un triángulo amoroso: español, subjuntivo, francés” en María Carmen Losada Aldrey, José F. Márquez Caneda y Tomás Eduardo Jiménez Juliá (coords.): Actas IX del Congreso Internacional de la ASELE, Santiago de Compostela: Universidad de Santiago de Compostela, 537-544.

Elıas, Vanessa, Valentyna Filımonova y Andrea Mojedano, 2014: "Prescription vs. praxis: The evolution of Spanish imperfect subjunctive", New Ways of Analyzing Variation 43.

FERnÁndez, Jesús, 1990: "Apuntes para la enseñanza del subjuntivo a anglohablantes" en Actas del Primer Congreso Nacional de ASELE, 21-31.

Gılı GaYA, Samuel, 1983: Curso superior de sintaxis española, Barcelona: Colton Book Imports.

Guzmán Naranjo, Matías, 2016: "The se-ra Alternation in Spanish Subjunctive”, Corpus Linguistics and Linguistic Theory 13 (1), 97-134. 
LARA, Luis Fernando, 2006: Curso de lexicología, México: El Colegio de México.

LLoRet, María-Rosa, 2011: "La alomorfía en la Teoría de la Optimidad” en Teoría morfológica y morfología del español, Madrid: Servicio de Publicaciones de la Universidad Autónoma de Madrid [http://www.uv.es/foncat, núm. 30, fecha de consulta: 10 de agosto de 2016].

LundH, Lars Gunnar, y Sylwia Czyzykow-CZarnocka, 2001: "Priming of the emotional stroop effect by a schema questionnaire. An experimental study of test order", Cognitive Therapy and Research 25 (3), 281-289.

Müller, Charles, 1973: Estadística lingüística, Madrid: Gredos.

PaGAno, Robert, 2012: Understanding statistics in the behavioral sciences, USA: Wadsworth.

Real Academia Española (RAE), 2010: Nueva gramática de la lengua española. Manual, Madrid: Espasa.

Rojo, Guillermo, 2008: "De nuevo sobre la frecuencia de las formas Ilegara y llegase” en Jörn AlBRECHT y Frank HARSLEm (eds.): Heidelberger Spätlese: Ausgewählte Tropfen aus verschiedenen Lagen der spanischen Sprach- und Übersetzungswissenschaft. Festschrift für Nelson Cartagena, Bonn: Romanistischer Verlag, 161-182.

SCHWENTER, Scott, 2013: "Strength of priming and the maintenance of variation in the Spanish past subjunctive", NWAV 42.

WILson, Joseph Michael, 1983: The -ra and -se Verb Forms in Mexico: A Diachronic Examination from Non-literary Sources. Tesis de doctorado, Universidad de Massachusetts. 